\title{
Hauttestung auf Nahrungsmittelallergie nach Allergenkarenz
}

\section{Skin Tests for Food Allergy After a Period of Allergen Avoidance}

Autoren

Institute

\section{Bayerl' ${ }^{1}$, A. Zimpfer ${ }^{2}$}

Klinik für Dermatologie und Allergologie, HSK, Wilhelm Freseniusklinik,

Lehrkrankenhaus der Universität Mainz, Wiesbaden

2 Klinik für Dermatologie, Venerologie und Allergologie, Universitätsklinik, Mannheim

\section{Bibliografie}

DOI $10.1055 / \mathrm{s}-2007-995373$

Akt Dermatol 2007; 33:

472-474 @ Georg Thieme

Verlag KG Stuttgart · New York ISSN 0340-2541

Korrespondenzadresse

Prof. Dr. med.

\section{Christiane Bayerl}

Klinik für Dermatologie und Allergologie HSK, Wilhelm Freseniusklinik Lehrkrankenhaus der Universität Mainz Aukammallee 39 65191 Wiesbaden christiane.bayerl@ hsk-wiesbaden.de

\section{Zusammenfassung $\nabla$}

Einige Allergologen argumentieren, dass sich eine Nahrungsmittelallergie nach Jahren der Allergenkarenz verliert. Patienten mit schwerer Nahrungsmittelallergie sind zurückhaltend gegenüber einem Plazebo kontrollierten oralen Provokationstest. Dieser Test wäre aber notwendig, um die Entwicklung einer Toleranz zu bestätigen oder zu widerlegen. Wir haben das Fortbestehen positiver Hauttestungen auf Nahrungsmittel nach 3 Jahren der Allergenkarenz untersucht. 45 von 157 Patienten mit systemischen anaphylaktischen Reaktionen Grad III und IV stimmten einer erneuten Testung im Prick/Scratch-Test zu. 20 $(44,4 \%)$ der erneut Getesteten waren weiterhin positiv auf das bekannte Nahrungsmittelallergen,
$14(29,9 \%)$ waren noch auf einige, aber nicht auf alle der ehemaligen Allergene positiv und 11 $(31,1 \%)$ zeigten negative Testergebnisse auf das bekannte Nahrungsmittelallergen. Bei den Wiederholungstestungen fanden wir einen Verlust der Hauttestpositivität vor allem gegenüber Fischen, Krustentieren und exotischen Früchten, während die Testungen weiterhin positiv waren bei Gewürzen, Nüssen, Gemüse und regionalen Früchten. Das konsequente Meiden des Allergens bei Nahrungsmittelallergikern führt bei einem Drittel der Patienten zu negativen Hauttestergebnissen. Wir empfehlen danach die doppeltblinde, Plazebo-kontrollierte orale Nahrungsmittelprovokationstestung durchzuführen, um mit letzter Sicherheit nachzuweisen, dass die Allergie sich zurückgebildet hat.

\section{Einleitung \\ $\nabla$}

Durchschnittlich 2\% der Erwachsenen leiden unter Nahrungsmittelunverträglichkeiten, die durch eine Vielzahl immunologischer Mechanismen ausgelöst werden, IgE-vermittelte oder nicht-IgEvermittelte Intoleranzreaktionen unterschiedlicher Art. Bei den IgE-vermittelten Reaktionen sprechen einige Autoren von einem Verlust der Nahrungsmittelallergie nach Jahren der Allergenkarenz [1]. Eine Rückbildung der Sensitivität über die Zeit ist bei Kindern bei weitem häufiger als bei Erwachsenen und findet sich bei manchen Nahrungsmitteln häufiger als bei anderen. Allergien gegenüber Kuhmilch und Ei verlieren sich üblicherweise früh im Leben bis zum 3. bzw. 5. Lebensjahr [2]. Bei Kindern und Erwachsenen wird die Ausbildung einer Toleranz nach vorausgegangener Allergie durch einen doppel-blinden, Plazebo-kontrollierten oralen Nahrungsmittelprovokationstest bewiesen (DBPCFC). Patienten mit schweren anaphylaktischen Reaktionen Grad III oder IV nach Ring und Messmer [3] werden einem Provokationstest zwar zustimmen, gehen damit jedoch ein hohes Risiko einer erneuten anaphylaktischen Reaktion ein. Es wäre daher wünschenswert, eine risikoarme Methode, den Hauttest, vorzuschalten.

\section{Methods}

$\nabla$

Um das Fortbestehen oder den Verlust positiver Hauttestergebnisse (Prick/Scratch-Test-Reaktionen) auf Nahrungsmittel zu überprüfen, wurden 157 Erwachsene mit einer systemischen anaphylaktischen Reaktion Grad III, IV (gemäß Ring and Messmer [3]) retrospektiv aus den Patientenakten erfasst, mittels Briefen kontaktiert und gebeten, sich zu einer erneuten Testung nach Allergenkarenz vorzustellen. Durch detaillierte Erhebung der Patientengeschichte, körperlicher Untersuchung und Labortestungen wurden Differenzialdiagnosen zu einer anaphylaktischen 


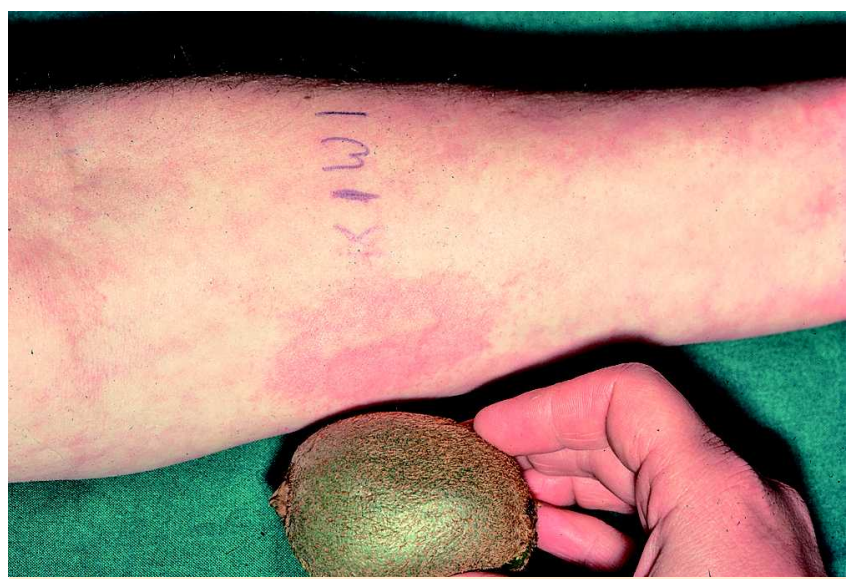

Abb. 1 Positive Scratch-Hauttestreaktion mit dem Fruchtfleisch einer Kiwi (ohne Schale) bei einer Patientin mit anaphylaktischer Reaktion Grad III nach Kiwigenuß. Testung vor der Zeit der 3-jährigen Allergenkarenz.

Reaktion ausgeschlossen. 45 (28,7\%) von 157 Patienten mit anayphylaktischer Reaktion Grad III oder IV auf Nahrungsmittel stimmten zu, sich 3 Jahre nach dem ersten positiven Hauttest und der dazwischen liegenden Zeit der Allergenkarenz erneut testen zu lassen. Diese bestätigten, dass in der Zeit der Allergenkarenz keine anaphylaktischen Reaktionen auf das ehemals als Allergen definierte Nahrungsmittel mehr aufgetreten waren. Entsprechend wurden aus dieser Gruppe 15 (67\%) männliche und 30 (33\%) weibliche Nahrungsmittelallergiker erneut einer Hauttestung unterzogen. Die Hauttestungen wurden mit kommerziellen Extrakten (Allergopharma, Reinbek, Germany) an der Beugeseite des Unterarms und mit nativen Allergenen im Prick-zu-Prick- oder Scratch-Test durchgeführt (negative Kontrolle $\mathrm{NaCl} \mathrm{0,9 \%}$ und positive Kontrolle Histamin) ( $\bullet$ Abb. 1). Zusätzlich wurden Daten zum gleichzeitigen Auftreten von Hautreaktionen auf Aeroallergene gesammelt. Die Bestimmung des spezifischen IgEs wurde nicht durchgeführt.

\section{Ergebnisse \\ $\nabla$}

Kreuzreaktionen oder gleichzeitig auftretende Reaktionen gegenüber Pollen zeigten sich im Hauttest bei $84 \%$, gegenüber Hausstaubmilbe bei $64 \%$ und gegenüber Latex bei $13 \% .20$ $(44,4 \%)$ der erneut getesteten Patienten waren weiterhin auf dasselbe Allergen wie zuvor im Hauttest positiv, 14 (29,9\%) waren positiv nicht mehr gegen alle, aber gegen einige der vorherigen Allergene, und 11 (31,1\%) zeigten ein negatives Hauttestergebnis gegenüber dem vorherigen Allergen ( $\bullet$ Tab. 1). In der Wiedertestung war ein Verlust der positiven Reaktion vor allem gegenüber Fischen, Krustentieren und exotischen Früchten zu finden, wohingegen der Hauttest weiterhin positiv blieb, besonders für Gewürze $(n=10)$, Nüsse $(n=21)$, Gemüse $(n=7)$ und regionale Früchte $(n=6)$; einige Patienten zeigten positive Testergebnisse auf zwei Nahrungsmittel.

\section{Diskussion}

Unverträglichkeitsreaktionen gegenüber Nahrungsmitteln werden oft von Patientenseite vermutet, lassen sich aber bei Hauttestungen und Provokationstestungen meist nicht beweisen.
Tab. 1 Negativer Hauttest (Prick/Scratch-Test) nach Allergenkarenz über 3 Jahre bei Patienten, die nur gegen ein Nahrungsmittel anaphylaktisch reagiert hatten

\begin{tabular}{|c|l|}
\hline $\begin{array}{l}\text { Patientennummer } \\
(\mathbf{n}=\mathbf{4 5})\end{array}$ & $\begin{array}{l}\text { Negativer Wiederholungstest nach } \\
\text { Allergenkarenz gegenüber }\end{array}$ \\
\hline 1 & Forelle \\
\hline 7 & Shrimps \\
\hline 10 & Kakao \\
\hline 11 & Scholle \\
\hline 17 & Eigelb \\
\hline 22 & Avokado \\
\hline 33 & Curry-Gewürzmischung \\
\hline 39 & Forelle \\
\hline 42 & Sellerie \\
\hline 43 & Lachs \\
\hline 44 & Kiwi \\
\hline
\end{tabular}

Der doppelt-blinde, Plazebo-kontrollierte orale Nahrungsmittelprovokationstest (DBPCFC) ist eine rigorose Nachweismethode, wird aber mittlerweile in der Medizin als Maßnahme akzeptiert, um die Notwendigkeit von Karenzdiäten bei Nahrungsmittelanaphylaxie zu verifizieren. Bei Patienten, die bereits eine anaphylaktische Reaktion im Leben erlitten haben, wird diese Methode mit Bedenken betrachtet. Das Einverständnis zu einem DBPCFC wird nicht so leicht erteilt wie zu einer Hauttestung. Wir haben unsere Studie auf Patienten gestützt, die initial eine positive Reaktion auf ein Nahrungsmittel hatten, das eine anaphylaktische Reaktion ausgelöst hatte. Nach einer Zeit der Allergenkarenz haben wir erneute Hauttestungen durchgeführt.

Generell haben negative Hauttestungen auf Nahrungsmittel einen hohen prädiktiven Wert einen IgE-mediierten Mechanismus der Unverträglichkeit auszuschließen. Aufgrund des Schweregrades der systemischen Reaktion Grad III und IV wurde in unserer Patientengruppe keine Nahrungsmittelprovokation durchgeführt. Bei der eosinophilen Ösophagitis haben sich Hauttestungen als nützlich erwiesen, um potenzielle Nahrungsmittelallergene zu identifizieren; dies waren zumeist Milch und Ei. Meiden führt zu einem Verschwinden der Symptome bei $69 \%$ der Patienten [4].

Bis vor kurzem wurde noch davon ausgegangen, dass eine Fischallergie lebenslang persistiert. Dagegen gab es eine Publikation, die von einem Verschwinden einer IgE-mediierten, ursprünglich im Hauttest positiven Fisch-Anaphylaxie auf Heilbutt berichtete. Nach Allergenkarenz wurde die Toleranz durch einen offenen Provokationstest mit Heilbutt belegt [5]. Auch unsere Ergebnisse geben Hinweise darauf, dass eine Fischallergie sich verlieren kann. Daher sollten Allergologen den allergologischen Ist-Zustand bei Ihren Patienten mit Fischallergie reevaluieren.

Interessanterweise wird ein Verschwinden der in den USA häufigen Erdnussallergie bei einigen Kindern mit Urtikaria oder mit Verschlechterung der Neurodermitis durch Erdnüsse berichtet. Dagegen entwickelte kein einziges Kind mit einer Anaphylaxie durch Erdnüsse eine Toleranz [6]. Offensichtlich sind einschneidende immunologische Ereignisse notwendig, um eine Toleranz zu induzieren. Dies wurde in einer Studie über einen Jungen gezeigt, der seine Erdnussallergie nach Knochenmarkstransplantation wegen primärer Immundefizienz verlor [7]. Die Wahrscheinlichkeit aus einer Erdnussallergie „herauszuwachsen“ wird auf $50 \%$ geschätzt, wobei Rückfälle beschrieben sind wenn auch selten [8]. In unserer Studie reagierten alle Patienten mit Erdnussallergie $(n=2)$ weiterhin positiv im Hauttest. 
Die Induktion einer spezifischen oralen Toleranz durch orale Hyposensibilisierung bei Kindern mit Milch- und Eiallergie ist eine Chance, aber dennoch sind nach Toleranzinduktion bei erneuter Gabe systemische Reaktionen aufgetreten $[9,10]$. Es ist vorrangig die Allergenkarenz, die als beste Prävention einer Anaphylaxie angesehen wird, wenn das Allergen identifiziert ist. Gemäß unseren Daten führt das Meiden des Allergens bei Nahrungsmittelallergie bei einem Drittel der Patienten zu negativen Hauttestergebnissen. Dies deckt sich fast mit einer Quelle, bei der nach einem Jahr Allergenkarenz und DBPCFC letztlich 38\% der Nahrungsmittel toleriert wurden - jedoch wurden zuvor keine Hauttestungen durchgeführt. Der Autor diskutiert mit Recht, dass der Erfolg der Eliminationsdiät von der Art des Nahrungsmittels abhängt und der Möglichkeit, das Allergen vollkommen zu meiden [11].

Unsere Studie bietet weiterhin Hinweise, dass eine Fischallergie keineswegs lebenslang zu persistieren scheint. Wir empfehlen daher, bei Patienten, die konsequente Allergenkarenz betrieben haben, erneute Hauttestung durchzuführen und dann den DBPCFC anzuschließen.

\section{Abstract}

\section{Skin Tests for Food Allergy After a Period of Allergen Avoidance}

$\nabla$

Some authors argue that food allergy will be lost after years of allergen avoidance. Patients with severe systemic anaphylactic reactions are reluctant to consent to a double blind placebo controlled oral provocation test to prove or disprove tolerance. We studied the persistence of positive skin test results for food allergies after 3 years of allergen avoidance. 45 out of 157 patients with systemic anaphylactic reactions grade III and IV consented to be retested by prick/scratch test. 20 (44.4\%) of the retested patients continued to test positive to the same food allergen as before, $14(29.9 \%)$ tested positive not to all, but to some of the former allergens and 11 (31.1\%) showed a negative skin test to the former allergen. In the repeat tests we detected a loss of allergic skin test reactions primarily towards the allergens found in fish, crustaceans and exotic fruits, whereas the skin test continued to be positive especially for spices, nuts, vegetables and regional fruits. Allergen avoidance in food allergy leads to a negative skin test in about one third of patients. We recommend the performance of double-blind, placebo controlled food challenges in order to prove conclusively that the allergy has been lost.

\section{Literatur}

1 Wüthrich B, Hofer T. Nahrungsmittelallergien. III: Therapie: Eliminationsdiät, symptomatische medikamentöse Prophylaxe und spezifische Hyposensibilisierung. Schweiz Med Wschr 1986; 116: $1446-$ 1449

2 Hourihane JO, Roberts SA, Warner JO. Resolution of peanut allergy: case-control study. BMJ 1998; 316: 1271 - 1275

3 Ring J, Messmer K. Incidence and severity of anaphylactoid reactions to colloid volume substitutes. Lancet 1977; 26: 466-469

4 Spergel JM, Beasoleil JL, Mascarenhas M, Liacouras CA. The use of skin prick tests and patch tests to identify causative foods in eosinophilic esophagitis. J Allergy Clin Immunol 2002; 109: 363- 368

5 Solensky $R$. Resolution of fish allergy: a case report. Ann Allergy Asthma Immunol 2003; 91: 411 - 412

6 Spergel JM, Beausoleil JL, Pawlowski NA. Resolution of childhood peanut allergy. Ann Allergy Asthma Immunol 2000; 85: 473-476

7 Hourihane JO, Rhodes HL, Jones AM, Veys P, Connett GJ. Resolution of peanut allergy following bone marrow transplantation for primary immunodeficiency. Allergy 2005; 60: 536- 537

8 Fleischer DM, Conover-Walker MK, Christie L, Burks AW, Wood RA. The natural progression of peanut allergy: resolution and the possibility of recurrence. J Allergy Clin Immunol 2003; 112: 12 - 14

9 Rolinck-Werninghaus C, Staden U, Mehl A, Hamelmann E, Beyer K, Niggemann $B$. Specific tolerance induction with food in children: transient or persistent effect on food allergy? Allergy 2005; 60: $1320-$ 1322

10 Staden U, Rolinck-Werninghaus C, Brewe F, Wahn U, Niggemann B, Beyer $K$. Specific oral tolerance induction in food allergy in children: efficacy and clinical patterns of reaction. Allergy 2007; 62: 1261 - 1269

11 Pastorello EA, Stocchio L, Pravettoni V, Bigi A, Schilke ML, Incorvaia C, Zanussi $C$. Role of the elimination diet in adults with food allergy. J Allergy Clin Immunol 1989; 84: 475-483 